\title{
A New Adaptive Technique for Enhancement of Zone-2 Settings of Distance Relay
}

\author{
Avinash N. Sarwade ${ }^{1}$, Pradeep K. Katti ${ }^{2}$, Jayant G. Ghodekar ${ }^{3}$ \\ ${ }^{1}$ Dr. Babasaheb Ambedkar Technological University, Raigarh, India \\ ${ }^{2}$ Department of Electrical Engineering, Dr. Babasaheb Ambedkar Technological University, Raigarh, India \\ ${ }^{3}$ Government College of Engineering, Karad, India \\ Email: \{asarwade, pk_katti2003\}@yahoo.com
}

Received August 14, 2011; revised September 18, 2011; accepted September 30, 2011

\begin{abstract}
This paper presents a technique that increases the second-zone coverage of distance relays without causing overreach problems. The technique is based on the impedance seen by distance relays when faults are simulated on the reach of zone-1 relays. The approach checks the relay operation for the maximum as well as the minimum generation outputs of the power system. The proposed technique can be used for different reach setting for zone-1. Results show that it is possible to increase the coverage provided by zone-2 distance relays without causing coordination problems with the primary relays that protect the lines emanating from the remote bus. The proposed method is modified for use in an adaptive protection system. It is shown that further improvements can be achieved when settings are calculated using the proposed method and the prevailing system conditions. The proposed method and its adaptive version were applied to a part of the Power system. The simulation of the system is done by using PSCAD-EMTDC software. The simulated results are observed and compared with the conventional and proposed technique.
\end{abstract}

Keywords: Power Systems; Distance Protection; Zone-2 Settings and Adaptive Protection

\section{Introduction}

It is a general practice to use high-speed primary protection along with slower speed backup protection in power systems. The backup protection becomes effective only if the primary protection relays fail to isolate the fault. The coordination is achieved by providing sufficient time delays between the operations of primary and backup relays.

Several approaches are used to protect transmission lines; one of these consists of using distance relays. The zone-2 relays are set to reach a fixed percentage of the shortest line emanating from the remote bus. Coordination with the zone- 1 relays protecting the lines emanating from the remote bus is achieved by delaying the trip outputs. These delays are of the order of 0.4 to $0.5 \mathrm{~s}$ that subject the power system to substantial shock. This approach intuitively considers the infeeds from sources connected to the remote buses [1].

Many papers have been published on the settings of distance protection, and different computer-aided techniques have been proposed for this purpose. [2] These techniques, in general, can be classified as based on: 1) topological analysis; 2) optimization theory; and 3) artificial intelligence [3,4]. Additionally, these techniques have been implemented as offline and online. The offline settings can only consider limited network states. The online settings (or adaptive settings), on the other hand, respond to the changing system conditions and adapt the relay settings according to the new conditions [5]. One technique for settings of transmission system distance protection based on an intelligent analysis of events and their consequences which include not only the zone reaches and zone time delays, as in the existing approaches, but also the optimal impedance operating characteristics, the fault detection settings, and the required relay sensitivities in terms of voltage and current [6]. Adaptive setting procedure is applied to overcome infeed, outfeed, arc resistance, load encroachment problems using the information received by the communication system and applying different logics [7].

This paper presents a mathematical approach that uses fault studies to determine the settings of the zone- 2 relays, taking into account the infeeds from all sources including those connected to the remote buses. The worst-case scenario is used for determining the settings because the contributions from remote sources are different for different operating conditions. The proposed method increases the percentage of line covered by zone-2 relays in comparison to the conventional method. 
With the deployment of microprocessor-based relays having communication capabilities, it is now possible to develope and implement adaptive protection systems. In these systems the relay settings can be automatically adjusted for making the protection more attuned to the prevailing system conditions. Therefore the settings of zone- 2 relays can be changed in order to cover higher percentage of the remote lines. Application of the proposed method for calculating zone-2 settings in an adaptive protection system is investigated. The method is modified so that the settings are calculated based on the prevailing system conditions and not the worst-case scenario as is done in non-adaptive protection systems. The proposed method and its adaptive version were applied to an existing power system and numbers of simulation studies are conducted and the results are reported in this paper.

To demonstrate and simulate the part of the power system for zone-2 setting of distance relay by conventional, proposed and adaptive method the PSCAD/EMTC software is used [8].

\section{Distance Protection and Its Settings}

Distance Relaying belongs to the principle of ratio comparison. The ratio is between voltage and current, which in turn produces impedance. The impedance is proportional to the distance in transmission lines, hence the distance relaying designation for the principle. This principle is primarily used for protection of high voltage transmission lines.

Under normal circumstances, the three-zone protection scheme of distance relay provides both primary and backup protection to the primary line, for all phase faults. Thus, defining the reach of all the zones along with the time delay associated with each zone can completely model a distance relay. The time delay of the zone 2 \& zone 3 has to be calculated such that, all faults have to be cleared within a maximum allowable time delay. However some delay in time is required to be given for the sequential breaker action to be coordinated. This time is called the minimum time delay. Generally, minimum allowable time delay is $0.3 \mathrm{sec}$. While the maximum value is $4 \mathrm{sec}$.

\subsection{Traditional Approach for Zone-2 Settings of for a Radial System}

Typically, First zone is set to reach $80 \%$ - $90 \%$ of the positive-sequence impedance of the line and the second-zone relays are set to reach $40 \%$ - 50\% into the next adjacent line having the lowest positive sequence impedance. Consider the system of Figure 1.

The impedance setting of the zone- 2 relay provided at bus $A$ is

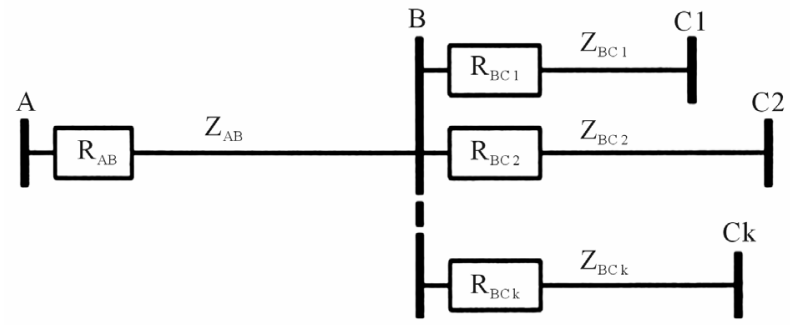

Figure 1. Radial transmission system protected by distance relays.

$$
Z-2\left(R_{A B}\right)=Z_{A B}+0.5 \times \operatorname{Minimum}\left(Z_{B C i}\right)
$$

In this equation,

$Z-2\left(R_{A B}\right)$ is the impedance setting for second-zone of relay $R_{A B}$;

$Z_{A B}$ is positive sequence impedance of the protected line $A-B$;

$Z_{B C i}$ is the positive sequence impedance of the shortest remote line $B-C i$.

$i$ equal to $1,2, \cdots, k$; $k$ is the total no. of primary relays.

\subsection{Part of the System under Consideration}

This criterion of Equation is used to define the setting of relay $R_{26}$ shown in the Figure 2 . The positive sequence impedances of lines are given in per unit values. The zone- 2 setting of relay $R_{26}$ is determined in this case. The line with the lowest impedance out of the remote line (other than the protected line) is the line from Bus B to Bus C. Substituting these values in Equation (1) provides the following setting for the zone-2 element.

$$
\begin{aligned}
Z-2\left(R_{26}\right)= & 0.03299+j 13586+0.50 \\
& \times(0.00446+j 0.01803) \\
Z-2\left(R_{26}\right)= & 0.14909 \angle 76.33 \mathrm{pu}
\end{aligned}
$$

\section{Zone-2 Settings by Proposed Method}

The proposed technique calculates the impedances seen by the zone- 2 relays when faults are on the lines emanating from the remote bus. The impedances are calculated for maximum and minimum outputs of generation sources. First-level contingencies are considered as well. The least apparent impedance calculated in these scenarios is used to determine the relay settings. This approach increases the reach of the zone- 2 relays without interfering with the operation of primary relays.

The proposed technique, described with reference to the system of Figure 3, consists of the following steps for setting the zone-2 element of relay $R_{B K}$.

1) Set the system to have maximum generation output.

2) Remove the line $B-C j$ from the system.

3) Simulate a three-phase fault (Fi) at the end of zone-1 


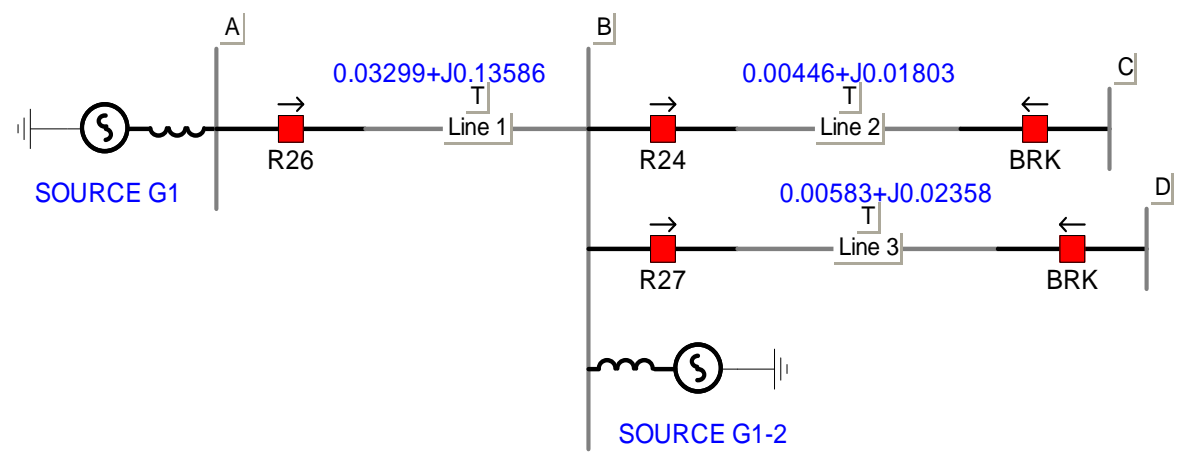

Figure 2. Part of a power system.

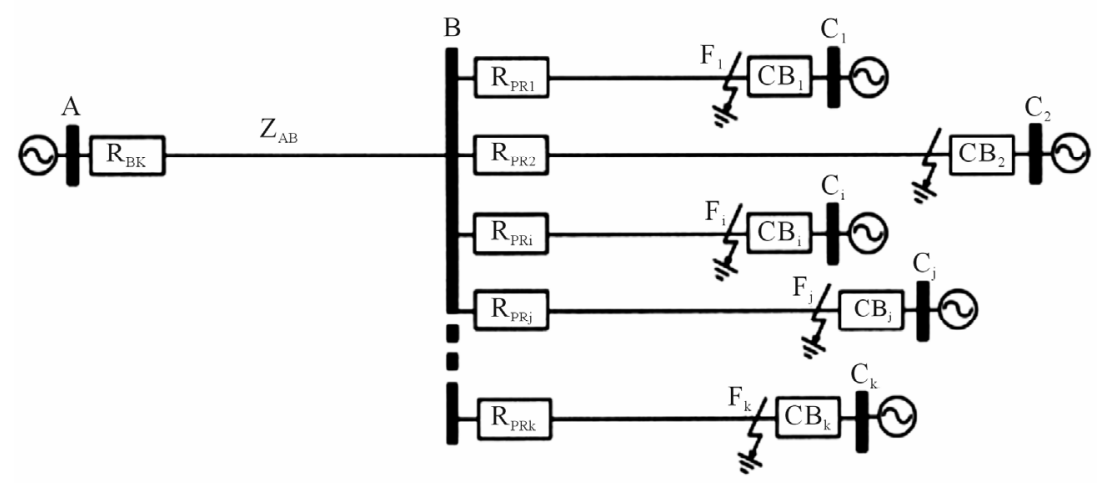

Figure 3. Primary relays and fault locations selected for determining zone-2 settings.

of line $B-C_{i}$ with the remote end breaker $C B i$ open ( $i$ is not equal to $j$ ).

4) Determine the setting of zone-2 element of relay $R_{B K}$ by using the apparent impedance seen by the relay $R_{B K}$ for fault $F i$ at and the following equation:

$$
Z-2\left(R_{B K}, R_{P R i}\right)=Z_{A B}+K 2 \times\left[Z_{A}\left(R_{B K}, F_{i}\right)-Z_{A B}\right)
$$

where

$R_{B K}$ backup (zone-2) relay whose setting is to be determined;

$R_{P R i}$ primary (zone-1) relay protecting line $B-C i$;

$Z_{A B}$ positive sequence impedance of the line $A-B$;

$K 2$ fraction of the remote lines protected by the zone- 2 relay [this value is $5 \%$ less than the percentage of line covered by primary (zone-1) (i.e. $\left.R_{P r i}\right)$ ];

$Z_{A}\left(R_{B K}, F_{i}\right)$ apparent impedance seen by the relay for fault Fi;

$Z-2\left(R_{B K}, R_{P R i}\right)$ possible setting of the zone-2 element of the relay $R_{B K}$.

However, if the apparent impedance seen by relay for fault is infinite (due to non presence of a source behind the relay), then $Z-2\left(R_{B K}, R_{P R i}\right)$ is set to $1.20 \times R_{B K}$.

5) Repeat steps 3 and 4 for faults at end of zone- 1 of other lines (fault at one line at a time).

6) Put the removed $B-C j$ back into service.

7) Repeat steps 3 to 6 with the remaining lines or other elements (transformers, generators, etc.) connected to bus
B taken out of service (removing one line or element at a time).

8) Select the least of the possible settings of the zone-2 relay calculated in steps 1 to 7 . Also, identify the remote removed line that generated this least setting.

9) Simulate a fault at the reach of the zone- 1 element of primary relay $R_{P r i}, \quad i=1,2,3, \cdots, k$, and all buses other than $B$. All circuit breakers are closed and the line, identified in step 8, is removed. Determine the seen impedance by the relay $R_{B K}$.

10) Compare the seen impedance by the relay $R_{B K}$ for the faults simulated in step 9 with the possible setting determined in step 8 to determine if the zone-2 element of the relay $R_{B K}$ would operate for these faults.

11 ) If the relay operates in step 10 , reduce the zone- 2 setting to reach the $90 \%$ of the smallest fault impedance, determined in step 9 seen by the relay $R_{B K}$.

12) Repeat steps 1 to 11 when generation output is minimum. The smaller of the zone-2 settings determined for maximum and minimum generation output is the final zone-2 setting for the relay $R_{B K}$.

This method was applied to the system shown in Figure 2, considering the system to have maximum generation output. Table 1 shows the possible setting of zone- 2 element of relay $R_{26}$ when three-phase faults occur at the end of zone- 1 of the lines emanating from the remote bus and single contingencies are simulated. The smallest 
zone-2 setting $(0.16959 \angle 85.44$ p.u.) for the relay happens when line 3 is removed, and the fault is on line 2 . Apparent impedance seen by zone-2 element of distance relay $R_{26}$, when the faults were simulated at the end of zone- 1 of primary relays and line 3 is removed, are given in Table 2. These fault impedances seen by the relay $R_{26}$ are bigger than its zone-2 setting selected from Table 1. Therefore, it is not necessary to change this setting.

Table 3 shows the possible setting of zone-2 relay when the system has minimum generation output. The smallest zone-2 setting ( $0.180602 \angle 85.42$ p.u.) for the relay happens, when line 3 is removed and the fault is on line 2 . The apparent impedance seen by zone- 2 element of distance relay $R_{26}$, when the faults were simulated at the end of zone- 1 of primary relays and line 3 is removed, are given in Table 4.

These impedances seen by the relay $R_{26}$ are bigger than its zone-2 setting selected from Table 3 . Therefore, it is not necessary to change this setting. This means that the zone-2 setting for relay is $0.16959 \angle 85.44$ p.u.

\section{Adaptive Settings of Relay}

The performance of the proposed method can be further enhanced if zone-2 settings can be made adaptive to the prevailing power system conditions. This would mean that the system condition should be monitored and new zone- 2 settings should be calculated in response to any changes in the operating conditions. The new settings are then communicated to the relays. The relay settings are, therefore, always attuned to the prevailing system conditions. This adaptive setting technique is described in this section $[9,10]$.

\section{Adaptive Settings Method for a Radial System}

This method is primarily similar to the non-adaptive method except that impedances need not be calculated for maximum and minimum levels of generation and no contingencies need to be considered. The seen impedances are calculated for the existing conditions. This means that the setting for zone-2 element of distance relay in adaptive protection system is determined by calculating the apparent impedance seen by zone- 2 relays when faults are simulated on the lines emanating from the remote bus considering the prevailing operating conditions of the power system. The adaptive setting method for zone-2 relays is described with reference to the system of Figure 3 and consists of the following steps for determining the zone-2 setting of the relay $R_{B K}$ [11].

1) Obtain the current operating condition of the power system (i.e., topology, power flow, and generation) [7]. Simulate a three-phase fault at the end of zone-1 of line $B$ - $C i$ with the remote end breaker $C B i$ open.

2) Determine the setting of zone-2 element of relay
Table 1. Possible zone-2 settings calculated for relay $\boldsymbol{R}_{26}$ on maximum generation.

\begin{tabular}{cccc}
\hline $\begin{array}{c}\text { Fault on the } \\
\text { end of zone-1 }\end{array}$ & Line-2 removed & Line-3 removed & $\begin{array}{c}\text { Source } \\
\text { G1-2 removed }\end{array}$ \\
\hline Line-2 & Line Out & $\mathbf{0 . 1 6 9 5 9} \angle \mathbf{8 5 . 4 4}$ & $0.21799 \angle 85.40$ \\
Line-3 & $0.17819 \angle 85.38$ & Line Out & $0.25138 \angle 85.27$ \\
\hline
\end{tabular}

Table 2. Fault impedance seen by the relay $R_{26}$ with faults at the end of zone-1 of each line.

\begin{tabular}{cc}
\hline Fault on the end of zone-1 & Max. Generation \& Line 3 removed \\
\hline Line-2 & Line Out \\
Line-3 & $0.19254 \angle 85.32$ \\
\hline
\end{tabular}

Table 3. Possible zone-2 settings calculated for relay $R_{26}$ on min generation.

\begin{tabular}{cccc}
\hline $\begin{array}{c}\text { Fault on the } \\
\text { end of zone-1 }\end{array}$ & Line-2 removed & Line-3 removed & $\begin{array}{c}\text { Source } \\
\text { G-12 removed }\end{array}$ \\
\hline Line-2 & Line Out & $\mathbf{0 . 1 8 0 6 0} \angle \mathbf{8 5 . 4 2}$ & $0.26820 \angle 85.80$ \\
Line-3 & $0.19254 \angle 85.32$ & Line Out & $0.32573 \angle 85.82$ \\
\hline
\end{tabular}

Table 4. Fault impedance seen by the relay $\boldsymbol{R}_{26}$ with faults at the end of zone-1 of each line.

\begin{tabular}{cc}
\hline Fault on the end of zone- 1 & Min Generation \& Line 3 removed \\
\hline Line-2 & Line Out \\
Line-3 & $0.19254 \angle 85.32$ \\
\hline
\end{tabular}

$R_{\mathrm{BK}}$ by using the apparent impedance seen by the relay $R_{B K}$ for fault at and the following equation:

$$
Z-2\left(R_{B K}, R_{P R i}\right)=Z_{A B}+K 2 \times\left[Z_{A}\left(R_{B K}, F_{i}\right)-Z_{A B}\right)
$$

In this equation

$R_{B K}$ backup (zone-2) relay whose setting is to be determined;

$R_{P R i}$ primary (zone-1) relay protecting line $\mathrm{B}-\mathrm{Ci}$;

$Z_{A B}$ positive sequence impedance of the line $A-B$;

$K 2$ fraction of the remote lines protected by the zone-2 relay [this value is $5 \%$ less than the percentage of line covered by primary (zone-1) that is, $R_{P R I}$;

$Z_{A}\left(R_{B K}, F_{i}\right)$ apparent imp seen by the relay for fault $\mathrm{Fi}$;

$Z-2\left(R_{B K}, R_{P R i}\right)$ possible setting of the zone-2 element of the relay $R_{B K}$.

However, if the apparent impedance seen by relay for fault is infinite (due to nonpresence of a source behind the relay) then $Z-2\left(R_{B K}, R_{P R i}\right)$ is set to $1.20 \times Z_{A B}$.

3) Repeat steps 2 and 3 for faults at end of zone- 1 of other lines (fault at one line at a time).

4) Select the least of the possible settings of the zone-2 
relay calculated in steps 2 to 4 .

5) Simulate a fault at the reach of zone-1 element of primary relay, $R_{P R i}=1,2, \cdots, k$, and all buses other than $B$. All circuit breakers are closed. Determine the seen impedance by the relay $R_{B K}$.

6) Compare the seen impedance by the relay $R_{B K}$ for the faults simulated in step 6 with the possible setting determined in step 5 to determine if the zone- 2 element of $R_{B K}$ would operate for these faults.

7 ) If the relay operates in step 7, reduce the zone-2 setting to reach the $90 \%$ of the smallest fault impedance, determined in step 6 seen by the relay $R_{B K}$.

8) Repeat steps 1 to 8 for determining zone- 2 setting of the remaining relays in the system.

The adaptive setting method was applied to determine zone-2 setting of relay $R_{26}$ of the system shown in Figure 2. Initially, the maximum generation is considered as the operating condition of the power system that has been reported to the central control computer. Table 5 shows the possible setting of zone-2 element of relay $R_{26}$ when three-phase faults occur at the end of zone- 1 of the remote lines. The possible zone-2 setting (smaller) for relay $R_{26}$ is

$$
Z-2\left(R_{26}\right)=0.24776 \angle 85.5 \text { p.u. }
$$

The impedance seen by relay $R_{26}$ when faults occur at the end of zone- 1 that is set at $90 \%$ of the protected line (step 6) are shown in Table 6. In this case, the impedance seen by relay $R_{26}$ is less than $0.24776 \angle 85.5$ p.u. when a fault is on line 2. Therefore, it is necessary to change this setting to apply step 8 of the method.

$$
\begin{aligned}
& Z-2\left(R_{26}\right)=0.9 \times 0.2207618 \angle 85.32 \\
& Z-2\left(R_{26}\right)=0.19868 \angle 85.32 \text { p.u. }
\end{aligned}
$$

Table 7 shows the possible setting of zone-2 element of relay $R_{26}$ Minimum Generation when three-phase faults occur at the end of zone- 1 of the remote lines. The possible zone-2 setting (smaller) for relay $R_{26}$ is

$$
Z-2\left(R_{26}\right)=0.275370 \angle 85.55 \text { p.u. }
$$

The impedance seen by relay $R_{26}$ when faults occur at the end of zone- 1 that is set at $90 \%$ of the protected line (step 6) are shown in Table 8. In this case, the impedance seen by relay $R_{26}$ is less than $0.275370 \angle 85.55$ p.u. when a fault is on line 12-11, Therefore, it is necessary to change this setting to apply step 8 of the method.

$$
\begin{aligned}
& Z-2\left(R_{26}\right)=0.9 \times 0.275370 \angle 85.55 \\
& Z-2\left(R_{26}\right)=0.247833 \angle 85.55 \text { p.u. }
\end{aligned}
$$

\section{Comparision of the Coverage Given by Different Methods}

The adaptive method for determining zone-2 settings provides higher coverage than nonadaptive version of the proposed method and the conventional method as shown in Figures 4 and 5. These figures and Table 9 show the zone-2 coverage by relay for the maximum and minimum generation conditions when zone- 2 of this relay is set by using the conventional method, nonadaptive, and adaptive version of the proposed method. It is clear from the figures that the proposed method (adaptive version) provides the most zone- 2 coverage for the lines.

\section{Conclusions}

The traditional methodology for setting distance relays provides minimum backup protection to remote lines. This approach works well when distance relays are used

Table 5. Possible zone-2 settings calculated for relay $\boldsymbol{R}_{26}$ on maximum generation.

\begin{tabular}{cc}
\hline Fault on the end of zone- 1 & Possible zone-2 settings (p.u.) \\
\hline Line-2 & $\mathbf{0 . 2 4 7 7 6} \angle \mathbf{8 5 . 5}$ \\
Line-3 & $0.292026 \angle 85.5$ \\
\hline
\end{tabular}

Table 6. Fault imp seen by the relay $R_{26}$ with the faults at the end of zone- 1 of each line.

\begin{tabular}{cr}
\hline Fault on the end of zone- 1 & Fault Impedance at Z-1 $\left(R_{P R i}\right)$ [p.u.] \\
\hline Line-2 & $\mathbf{0 . 2 2 0 7 6} \angle \mathbf{8 5 . 3 2}$ \\
Line-3 & $0.25396 \angle 85.45$ \\
\hline
\end{tabular}

Table 7. Possible zone-2 settings calculated for relay $\boldsymbol{R}_{26}$ on min generation.

\begin{tabular}{cc}
\hline Fault on the end of zone- 1 & Possible zone-2 settings (pu) \\
\hline Line-2 & $\mathbf{0 . 3 2 0 5 2 9} \angle \mathbf{8 5 . 6 2}$ \\
Line-3 & $0.39671 \angle 85.59$ \\
\hline
\end{tabular}

Table 8. Fault imp seen by the relay $R_{26}$ with the faults at the end of zone-1 of each line.

\begin{tabular}{cc}
\hline Fault on the end of zone-1 & $\begin{array}{c}\text { Fault Impedance at Z-1 }\left(R_{P R i}\right) \\
\text { [p.u.] }\end{array}$ \\
\hline Line-2 & $\mathbf{0 . 2 7 5 3 7 0 ~} \angle \mathbf{8 5 . 3 2}$ \\
Line-3 & $0.332499 \angle 85.45$ \\
\hline
\end{tabular}

Table 9. Zone-2 coverage by relay for the maximum and minimum generation conditions for conventional, non adaptive, and adaptive version of the proposed method.

\begin{tabular}{cccc}
\hline $\begin{array}{c}\text { Level of } \\
\text { Generation }\end{array}$ & $\begin{array}{c}\text { Conventional } \\
\text { Method (p.u.) }\end{array}$ & $\begin{array}{c}\text { Proposed Method } \\
\text { (p.u.) }\end{array}$ & $\begin{array}{c}\text { Adaptive Setting } \\
\text { Method (p.u.) }\end{array}$ \\
\hline Maximum & $0.14914 \angle 85.55$ & $0.16959 \angle 85.44$ & $0.19868 \angle 85.32$ \\
Minimum & $0.14914 \angle 85.55$ & $0.18060 \angle 85.42$ & $0.247833 \angle 85.55$ \\
\hline
\end{tabular}




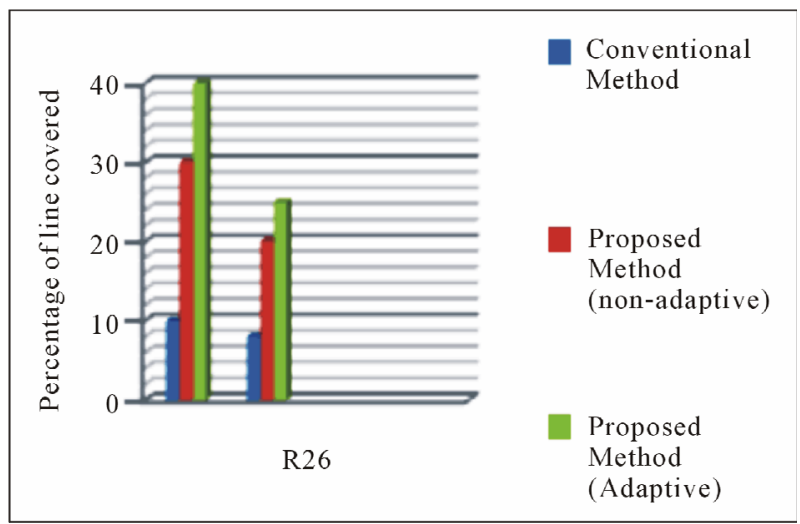

Figure 4. Percentage of the line covered for the maximum generation condition.

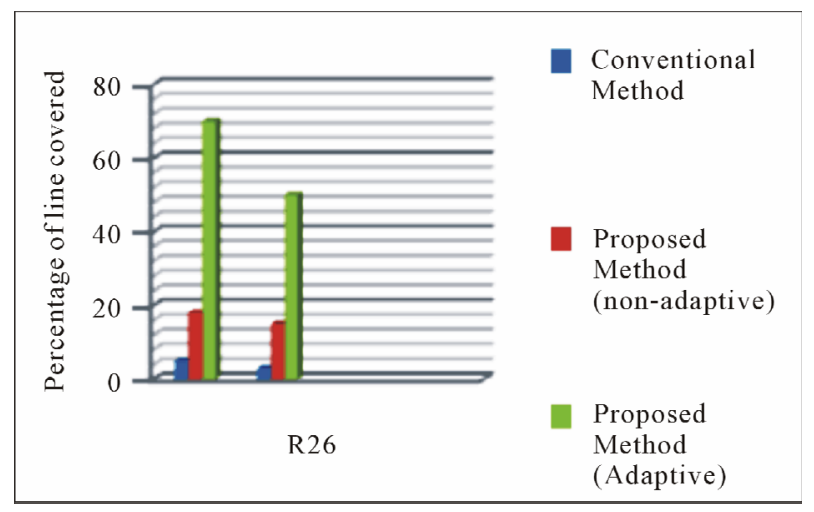

Figure 5. Percentage of the line covered for the minimum generation condition.

to protect radial lines in which there are no generation sources connected to the remote buses [12,13].

The proposed technique increases the reach of the zone-2 distance relays without affecting the security of the system. This is a useful feature that improves the protection during circuit breaker failures on the lines emanating from the remote bus especially on lines that are not provided with sophisticated protection schemes. Also, some faults that would have been cleared by the zone-3 elements previously will now be cleared by zone-2 relays. The time delays in these cases are substantially reduced. The proposed technique becomes important in cases where breaker failure schemes are not used.

This can be further improved if the proposed method can be made adaptive. This will require online monitoring of the power system.

It is concluded that both versions of the proposed method provide better line coverage than the conventional method but adaptive version further improves the coverage. It is suggested that in case of communication failure, non adaptive version of the proposed method can be used and it will provide better zone-2 coverage than the conventional method.
However better setting or protection of transmission line can be improved by using modified distance relay characteristics [14-17].

\section{REFERENCES}

[1] J. L. Blackburn, "Protective Relaying Principles and Applications.,” Marcel Dekker, New York, 1987.

[2] M. J. Damborg, R. Ramaswami, S. S. Venkata and J. Postforoosh, "Computer Aided Transmission Protection System Design,” IEEE Transactions on Power Apparatus and System, Vol. PAS-103, No. 1, 1984, pp. 51-59. doi:10.1109/TPAS.1984.318576

[3] M. H. Dwarakanath and L. Nowitz, “An Application of Linear Graph Theory for Coordination of Directional Overcurrent Relays,” Electric Power Problems: Mathematical Challenge, SIAM, Philadelphia, 1980, pp. 104114.

[4] M. S. Sachdev, T. S. Sidhu and B. K. Talukdar, "Topology Detection for Adaptive Protection of Distribution Networks," Proceedings of IEEE Energy Manage. Power Delivery, Singapore City, 21-23 November 1995, pp. 445-450.

[5] B. Chattopadhyay, M. S. Sachdev and T. S. Sidhu, “An On-Line Relay Coordination Algorithm for Adaptive Protection Using Linear Programming Technique,” IEEE Transactions on Power Delivery, Vol. 11, No. 1, 1996, pp. 165-173. doi:10.1109/61.484013

[6] K. El-Arroudi, G. Joós, D. T. McGillis and R. Brearley, "Comprehensive Transmission Distance Protection Settings Using an Intelligent-Based Analysis of Events and Consequences,” IEEE Transactions on Power Delivery, Vol. 20, No. 3, 2005, pp. 1817-1824. doi:10.1109/TPWRD.2005.848656

[7] M. I. Gilany, B.-E. Hasan and O. P. Malik, "The Egyptian Electricity Authority Strategy for Distance Relay Setting: Problems and Solutions," Electric Power System Research, Vol. 56, No. 2, 2000, pp. 89-94. doi:10.1016/S0378-7796(00)00093-6

[8] PSCAD/EMTDC 4.2.1, Manitoba HVDC Research Centre Inc., Manitoba, 2008.

[9] S. H. Horowitz, A. G. Phadke and J. S. Thorp, “Adaptive Transmission System Relaying,” IEEE Transactions on Power Delivery, Vol. 3, No. 4, 1988, pp. 1436-1445. doi:10.1109/61.193942

[10] G. D. Rockefeller, C. L. Wagner and J. R. Linders, “Adaptive Transmission Relaying Concepts for Improved Performance,” IEEE Transactions on Power Delivery, Vol. 3, No. 4, 1988, pp. 1446-1458. doi:10.1109/61.193943

[11] M. S. Sachdev and T. S. Sidhu, "A New Approach for Calculating Zone-2 Settings of Distance Relays and Its Use in Adaptive Protection System," IEEE Transactions on Power Delivery, Vol. 19, No. 1, 2004, pp. 70-77. doi:10.1109/TPWRD.2003.820202

[12] B. Chattopadhyay, M. S. Sachdev and T. S. Sidhu, "Protection of a Distribution Network-An Adaptive Approach,” Canadian Journal of Electrical and Computer 
Engineering, Vol. 19, No. 3, 1994, pp. 99-108.

[13] A. K. Jampala, S. S. Venkata and M. J. Damborg, “Adaptive Transmission Protection: Concepts and Computational Issues," IEEE Transactions on Power Delivery, Vol. 4, No. 1, 1989, pp. 177-185. doi:10.1109/61.19203

[14] A. N. Sarwade, P. K. Katti and J. G. Ghodekar, “Transmission Line Protection by Adaptive Digital Distance Relay," International Conference on Electrical Engineering, ICEE-2010, Penang, Vol. 62, 24-26 February 2010, pp. 1111-1115.

[15] A. N. Sarwade, P. K. Katti and J. G. Ghodekar, "Modified Distance Relay Characteristics for Dynamic Loading of Transmission Lines,” International Conference on Electrical Power and Energy System, Bhopal, 26-28 August 2010, pp. 493-499.

[16] A. N. Sarwade, P. K. Katti and J. G. Ghodekar, “Adaptive Solutions for Distance Relay Settings,” IEEE's 9th International Power and Energy Conference, Suntec, 27-29 October 2010, pp. 493-498.

[17] A. N. Sarwade, P. K. Katti and J. G. Ghodekar, “Advanced Modified Distance Relay Characteristics for Dynamic Loading," IEEE's 9th International Power and Energy Conference, Suntec, 27-29 October 2010, pp. 509-514. 